\title{
Electricity market clearing with improved dispatch of stochastic production
}

\author{
Morales González, Juan Miguel; Zugno, Marco; Pineda, Salvador; Pinson, Pierre
}

Published in:

European Journal of Operational Research

Link to article, DOI:

10.1016/j.ejor.2013.11.013

Publication date:

2014

Document Version

Early version, also known as pre-print

Link back to DTU Orbit

Citation (APA):

Morales González, J. M., Zugno, M., Pineda, S., \& Pinson, P. (2014). Electricity market clearing with improved dispatch of stochastic production. European Journal of Operational Research, 235(3), 765-774.

https://doi.org/10.1016/j.ejor.2013.11.013

\section{General rights}

Copyright and moral rights for the publications made accessible in the public portal are retained by the authors and/or other copyright owners and it is a condition of accessing publications that users recognise and abide by the legal requirements associated with these rights.

- Users may download and print one copy of any publication from the public portal for the purpose of private study or research.

- You may not further distribute the material or use it for any profit-making activity or commercial gain

- You may freely distribute the URL identifying the publication in the public portal

If you believe that this document breaches copyright please contact us providing details, and we will remove access to the work immediately and investigate your claim. 
Elsevier Editorial System(tm) for European Journal of Operational Research Manuscript Draft

Manuscript Number:

Title: Electricity Market Clearing With Improved Scheduling of Stochastic Production

Article Type: Innovative Application of OR

Section/Category: OR in energy

Keywords: OR in energy; electricity market; stochastic programming; electricity pricing; wind power; bilevel programming

Corresponding Author: Dr. Juan Miguel Morales, Ph.D.

Corresponding Author's Institution: Technical University of Denmark

First Author: Juan Miguel Morales, Ph.D.

Order of Authors: Juan Miguel Morales, Ph.D.; Marco Zugno, PhD student; Salvador Pineda, PhD; Pierre Pinson, $\mathrm{PhD}$

Abstract: In this paper, we consider an electricity market that consists of a day-ahead and a balancing settlement, and includes a number of stochastic producers. We first introduce two reference procedures for scheduling and pricing energy in the day-ahead market: on the one hand, a conventional network-constrained auction purely based on the least-cost merit order, where stochastic generation enters with its expected production and a low marginal cost; on the other, a counterfactual auction that also accounts for the projected balancing costs using stochastic programming. Although the stochastic clearing procedure attains higher market efficiency in expectation than the conventional day-ahead auction, it suffers from fundamental drawbacks with a view to its practical implementation. In particular, it requires flexible producers (those that make up for the lack or surplus of stochastic generation) to accept losses in some scenarios. Using a bilevel programming framework, we then show that the conventional auction, if combined with a suitable day-ahead dispatch of stochastic producers (generally different from their expected production), can substantially increase market efficiency and emulate the advantageous features of the stochastic optimization ideal, while avoiding its major pitfalls.

A two-node power system serves as both an illustrative example and a proof of concept. Finally, a more realistic case study highlights the main advantages of a smart day-ahead dispatch of stochastic producers. 
Dear Editor of European Journal of Operational Research,

Today's electricity market design is the result of adapting traditional practices, such as unit commitment, economic dispatch or contingency analysis, to a competitive structure. These practices were conceived in view of a generation mix mostly formed by dispatchable power plants, and are now to be revisited so that stochastic producers can enter the competition in a fair and efficient manner.

In this line, researchers have recently advocated a market-clearing mechanism that co-optimizes the forward (day-ahead) and the anticipated real-time energy dispatches using stochastic programming. Even though, ideally, this mechanism attains maximum market efficiency, it results in an energy-only market settlement that requires flexible producers to accept economic losses for some realizations of the stochastic production, which raises concerns on its practical applicability.

Starting from this point, this paper shows that, if stochastic production is conveniently scheduled in the day-ahead market, the conventional settlement of this market can notably approach the behavior of the stochastic ideal, while sidestepping its theoretical drawbacks. To this end, we construct a bilevel programming formulation that determines the optimal value of stochastic production that should be considered to clear the day-ahead market under the conventional settlement.

We firmly believe that this research work could positively contribute to your Journal. We thank you in advance for considering this manuscript.

Yours sincerely,

Juan Miguel Morales

Marco Zugno

Salvador Pineda

Pierre Pinson 
$\underline{\text { Highlights }}$

- Day-ahead markets should not be cleared considering the expected value of stochastic production

- The stochastic dispatch model maximizes market efficiency, but requires flexible producers to accept losses in some scenarios

- If stochastic generation is conveniently scheduled, the conventional dispatch approaches the stochastic ideal, while being revenue adequate per scenario

- We propose a bilevel programming problem to determine the optimal forward schedule of stochastic producers under the conventional settlement

- A two-bus example and a 24-bus case study illustrate the features of the improved scheduling of stochastic generation 
1

2

3

\title{
Electricity Market Clearing With Improved Scheduling of Stochastic Production
}

\author{
Juan M. Morales ${ }^{\mathrm{a}, *}$, Marco Zugno $^{\mathrm{b}}$, Salvador Pineda ${ }^{\mathrm{a}}$, Pierre Pinson ${ }^{\mathrm{b}}$ \\ ${ }^{a}$ Department of Electrical Engineering, Technical University of Denmark, Kgs. Lyngby, \\ Denmark \\ ${ }^{b}$ Department of Applied Mathematics and Computer Science, Technical University of \\ Denmark, Kgs. Lyngby, Denmark
}

\begin{abstract}
In this paper, we consider an electricity market that consists of a day-ahead and a balancing settlement, and includes a number of stochastic producers. We first introduce two reference procedures for scheduling and pricing energy in the day-ahead market: on the one hand, a conventional networkconstrained auction purely based on the least-cost merit order, where stochastic generation enters with its expected production and a low marginal cost; on the other, a counterfactual auction that also accounts for the projected balancing costs using stochastic programming. Although the stochastic clearing procedure attains higher market efficiency in expectation than the conventional day-ahead auction, it suffers from fundamental drawbacks with a view to its practical implementation. In particular, it requires flexible producers (those that make up for the lack or surplus of stochastic generation) to accept losses in some scenarios. Using a bilevel programming framework, we then show that the conventional auction, if combined with a suitable day-ahead dispatch of stochastic producers (generally different from their expected production), can substantially increase market efficiency and emulate the ad-
\end{abstract}

\footnotetext{
${ }^{*}$ Corresponding author. Phone: +4552759806

Email addresses: jmmgo@elektro.dtu.dk (Juan M. Morales), mazu@imm.dtu.dk (Marco Zugno), spmo@elektro.dtu.dk (Salvador Pineda), pp@imm.dtu.dk (Pierre Pinson)
} 
vantageous features of the stochastic optimization ideal, while avoiding its major pitfalls.

A two-node power system serves as both an illustrative example and a proof of concept. Finally, a more realistic case study highlights the main advantages of a smart day-ahead dispatch of stochastic producers.

Keywords: OR in energy, electricity market, stochastic programming, electricity pricing, wind power, bilevel programming

\section{Introduction}

The penetration of stochastic production in electric energy systems is notably increasing worldwide, primarily owing to a booming wind power industry. There is a broad consensus in the research community that today's electricity market designs are to be revisited so that stochastic producers can enter the competition in a fair and efficient manner.

In its most basic form, an electricity market consists of a forward (typically day-ahead) market and a balancing market. On the one hand, the day-ahead market is required to accommodate the generation from the inflexible power plants, i.e. from those generating units that need advance planning in order to efficiently and reliably set their production levels. On the other, the balancing market clears the energy deployed to maintain the constant balance of supply and demand over periods of time with finer resolution, commonly spanning from minutes to one hour. Being cleared shortly before real time, balancing markets allow the trade of energy between flexible firms, which can adjust their output quickly, and stochastic producers, whose generation is predictable only with limited accuracy at the day-ahead stage.

Conventionally the day-ahead and the balancing markets are settled independently. Furthermore, with respect to the participation of stochastic producers, the day-ahead market is typically cleared considering their expected production at a very low marginal cost (e.g., zero). The eventual energy adjustments needed to cope with the associated forecast errors are left then 
to the flexible units participating in the balancing market. Consequently, if this market is not provided with enough flexible capacity, balancing costs may escalate dramatically. It is expected that this problem becomes exacerbated as the penetration of stochastic production increases (Holttinen, 2005; Doherty \& O’Malley, 2005; Helman et al., 2010).

To face this challenge, two main solution strategies have been considered, namely:

1. To establish reserve markets, where flexible capacity is procured sufficiently in advance of energy delivery and then made available to the balancing market, where it is dispatched if needed. The reserve demand in these markets is exogenously specified by the Transmission System Operator, which opens up a number of different ad-hoc criteria, see e.g. Ela et al. (2011).

2. To clear the forward market using stochastic programming (Birge \& Louveaux, 2011), which allows modeling future balancing needs and costs in a probabilistic framework, thus yielding the day-ahead energy dispatch that minimizes the expected system operating costs. One of the major advantages of this approach is that it endogenously solves for the optimal amount of reserve capacity to be left to the balancing market, weighing the expected costs and benefits of such capacity (Galiana et al., 2005; Bouffard \& Galiana, 2008; Morales et al., 2009; Papavasiliou et al., 2011).

Ideally, the stochastic solution method attains maximum market efficiency (as it minimizes the expected system operating cost) and therefore, it is used here as a reference in this respect. For its practical application within a market environment, though, it must be first complemented with a set of prices and payments that make market participants satisfied with the resulting day-ahead dispatch. In this vein, Galiana et al. (2005) and Wong $\&$ Fuller (2007) define prices for both energy and reserve capacity. However, determining who should pay for such reserve and to which extent is still a 
major source of conflict and debate (Hogan, 2005).

In this paper, we follow the approach of Pritchard et al. (2010) and Morales et al. (2012), where the stochastic dispatch is supported by energy prices only. However, this approach is not without its problems either. Indeed, Morales et al. (2012) illustrate that the energy-only market settlement associated with the stochastic dispatch requires flexible producers to accept losses for some realizations of the stochastic production, which also raises concerns on its practical applicability.

Starting from this point, the objective of this paper is to show that, if cleared with an appropriate value of stochastic production, generally different from the expected value, the conventional settlement of the day-ahead market can notably approach the behavior of the ideal stochastic dispatch, while sidestepping its theoretical drawbacks. For this purpose, we construct a bilevel programming formulation that determines the optimal value of stochastic production that should be used to clear the day-ahead market under the conventional settlement.

The rest of this paper is organized as follows. Section 2 presents the conventional and stochastic dispatch models that we use as references in our work, and provides the mathematical insight to calculate the optimal day-ahead schedule of stochastic production under the conventional market settlement. Section 3 discusses results from a small example and a case study. More specifically, the example serves to illustrate the different dispatch models, which are subsequently compared and tested using a more realistic setup in the case study. Lastly, Section 4 concludes the paper.

\section{Dispatch Models}

Consider the sequence of a day-ahead and a balancing market. The dayahead market is cleared on day $d-1$ (e.g., by 10 am) and covers energy transactions for delivery on day $d$, typically on an hourly basis. The balancing market settles the energy imbalances with respect to the day-ahead produc- 
tion and consumption schedule. These imbalances are computed throughout day $d$, usually over time intervals ranging from minutes to 1 hour.

Let us begin by outlining a standard model for the dispatch of energy. This will serve to present the notation and provide a starting point for the developments of the rest of the paper. The setting will be an electric power system comprising a collection $N$ of nodes.

\subsection{Conventional Dispatch (ConvD)}

Let $p_{G}$ and $p_{W}$ denote the vectors of decisions on the day-ahead dispatch of conventional and stochastic producers, respectively. For simplicity and without loss of generality, the demand at each node $n$ of the system, $l_{n}$, is considered to be known with certainty. We also assume that power flows in the transmission network are determined by the vector $\delta^{0}$ of nodal voltage angles.

The conventional economic dispatch model (ConvDM) identifies the optimal schedule $\left(p_{G}^{*}, p_{W}^{*}\right)$ that minimizes day-ahead generating costs, $\mathcal{C}^{\mathrm{D}}\left(p_{G}, p_{W}\right)$, as follows:

$$
\begin{array}{ll}
\underset{p_{G}, p_{W}, \delta^{0}}{\operatorname{Minimize}} & \mathcal{C}^{\mathrm{D}}\left(p_{G}, p_{W}\right) \\
\text { s.t. } & h^{\mathrm{D}}\left(p_{G}, p_{W}, \delta^{0}\right)-l=0: \lambda^{\mathrm{D}}, \\
& g^{\mathrm{D}}\left(p_{G}, \delta^{0}\right) \leq 0, \\
& p_{W} \leq \widehat{W},
\end{array}
$$

where $\widehat{W}$ is the forecast vector of stochastic production. The equality constraints (1b) enforce the day-ahead balancing conditions, stating that the dispatch plus net power flow equals the demand at each node. The inequalities (1c) include upper and lower bounds to the dispatch of conventional producers and scheduled power flows, as well as declarations of non-negative variables. Constraints (1d) limit the day-ahead schedule of stochastic producers to their expected generation. 
The dispatch model (1) can be understood as a network-constrained auction that follows a least-cost merit-order principle, i.e., the cheapest generators are dispatched first. Consequently, because stochastic producers enter the market with very low or zero marginal cost, their dispatch up to the forecast mean $\widehat{W}$ is prioritized.

Notice that the vector of dual variables associated with constraint (1b), which is indicated in (1) by $\lambda^{\mathrm{D}}$, constitutes the vector of day-ahead locational marginal prices.

Once the optimal day-ahead schedule $\left(p_{G}^{*}, p_{W}^{*}\right)$ has been obtained from (1), the balancing market must deal with the energy imbalance caused by the stochastic production. Consider a specific realization vector of this production, denoted by $W_{\omega^{\prime}}$. The energy imbalance is then given by $W_{\omega^{\prime}}-p_{W}^{*}$, which represents a surplus of generation, if positive, or a shortage, if negative. To accommodate an excess of production, several actions may be taken, namely:

- To decrease the power production of flexible generating units. In market terms, this is equivalent to say that flexible producers repurchase a certain amount $r_{\omega^{\prime}}^{-}$of energy in the balancing market.

- To spill a part $W_{\omega^{\prime}}^{\text {spill }}$ of the stochastic production.

Similarly, to balance a deficit of generation, the following actions may be taken:

- To increase the power output of flexible units, which is equivalent to say that flexible producers sell an additional amount $r_{\omega^{\prime}}^{+}$of energy in the balancing market.

- To shed a portion $l_{\omega^{\prime}}^{\text {shed }}$ of the demand. This action is, in general, very costly, as the so-called value of lost load is normally very high.

It should be noticed that the previous decision vectors $r_{\omega^{\prime}}^{-}, r_{\omega^{\prime}}^{+}, W_{\omega^{\prime}}^{\text {spill }}$, and $l_{\omega^{\prime}}^{\text {shed }}$ have been intentionally augmented with the subscript $\omega^{\prime}$ to underline their 
implicit dependence on the specific realization $W_{\omega^{\prime}}$ of stochastic production. For ease of presentation, we group all these decision variables into one single vector $y_{\omega^{\prime}}$ (the notation introduced here will become relevant later on in the illustrative example of Section 3). Thus, the vector $y_{\omega^{\prime}}^{*}$ that minimizes the cost of balancing the energy deviation $W_{\omega^{\prime}}-p_{W}^{*}$ is solution to the following optimization problem:

$$
\begin{array}{ll}
\underset{y_{\omega^{\prime}}, \delta_{\omega^{\prime}}}{\operatorname{Minimize}} & \mathcal{C}^{\mathrm{B}}\left(y_{\omega^{\prime}}\right) \\
\text { s.t. } & h^{\mathrm{B}}\left(y_{\omega^{\prime}}, \delta_{\omega^{\prime}}, \delta^{0 *}\right)+W_{\omega^{\prime}}-p_{W}^{*}=0: \lambda_{\omega^{\prime}}^{\mathrm{B}}, \\
& g^{\mathrm{B}}\left(y_{\omega^{\prime}}, \delta_{\omega^{\prime}}, p_{G}^{*} ; W_{\omega^{\prime}}\right) \leq 0,
\end{array}
$$

where $\delta_{\omega^{\prime}}$ is the vector of nodal voltage angles at the balancing stage. The equality constraints $(2 \mathrm{~b})$ ensure that generating units and loads are redispatched so that the system remains in balance. The vector $\lambda_{\omega^{\prime}}^{\mathrm{B}}$ of dual variables associated with these constraints define the locational marginal prices at the balancing market. Similarly to (1c), the inequalities (2c) comprise upper and lower bounds on the re-dispatch of generating units, load shedding, wind spillage, actual power flows, and declarations of nonnegative variables.

If we now denote the optimal vector of balancing actions by $y_{\omega^{\prime}}^{*}$, the overall cost of operating the power system under the realization $W_{\omega^{\prime}}$ of stochastic production is given by $\mathcal{C}^{\mathrm{D}}\left(p_{G}^{*}, p_{W}^{*}\right)+\mathcal{C}^{\mathrm{B}}\left(y_{\omega^{\prime}}^{*}\right)$.

It is important to stress that both constraints $(2 \mathrm{~b})$ and $(2 \mathrm{c})$, and hence also the balancing costs $\mathcal{C}^{\mathrm{B}}\left(y_{\omega^{\prime}}\right)$, are dependent on the optimal day-ahead schedule $\left(p_{G}^{*}, p_{W}^{*}, \delta^{0 *}\right)$. Since the conventional dispatch model (1) is blind to such dependency, the market becomes more and more inefficient as the penetration of stochastic production increases. In this vein, the stochastic dispatch model presented next intends to capture precisely the interaction between day-ahead and balancing decisions. 


\subsection{Stochastic Dispatch (StochD)}

Consider that the electricity production from stochastic producers can be efficiently modeled by a finite set $\Omega$ of scenarios, each characterized by a vector of power values $W_{\omega}$ and a probability of occurrence $\pi_{\omega}$. It must hold that $\pi_{\omega} \geq 0$, for all $\omega \in \Omega$, and $\sum_{\omega \in \Omega} \pi_{\omega}=1$. The scenario set $\Omega$ is assumed to be available to the Transmission System Operator.

The stochastic dispatch model writes as follows:

$$
\begin{array}{ll}
\underset{p_{G}, p_{W}, \delta^{0} ; y_{\omega}, \delta_{\omega}, \forall \omega}{\operatorname{Minimize}} & \mathcal{C}^{\mathrm{D}}\left(p_{G}, p_{W}\right)+\mathbb{E}_{\omega}\left[\mathcal{C}^{\mathrm{B}}\left(y_{\omega}\right)\right] \\
\text { s.t. } & h^{\mathrm{D}}\left(p_{G}, p_{W}, \delta^{0}\right)-l=0: \lambda^{\mathrm{D}}, \\
& g^{\mathrm{D}}\left(p_{G}, \delta^{0}\right) \leq 0, \\
& p_{W} \leq \bar{W}, \\
& h^{\mathrm{B}}\left(y_{\omega}, \delta_{\omega}, \delta^{0}\right)+W_{\omega}-p_{W}=0, \quad \forall \omega \in \Omega, \\
& g^{\mathrm{B}}\left(y_{\omega}, \delta_{\omega}, p_{G} ; W_{\omega}\right) \leq 0, \quad \forall \omega \in \Omega,
\end{array}
$$

where $\bar{W}$ is the vector of capacities of stochastic producers and $\mathbb{E}_{\omega}[\cdot]$ is the expectation operator over the scenario set $\Omega$. Notice that, based on this set, the dispatch problem (3) explicitly models and thus anticipates the balancing operation of the power system by means of constraints (3e) and (3f) and the expectation of the balancing costs in the objective function (3a). This way, the stochastic programming problem (3) yields the day-ahead dispatch $\left(p_{G}^{*}, p_{W}^{*}\right)$ that maximizes market efficiency, provided that the scenario set $\Omega$ is properly constructed. As we shall see later, according to (3), flexible producers may be dispatched out of merit order in the day-ahead market to provide the power system with sufficient flexible capability to cope with the energy imbalances caused by stochastic producers in real time.

\subsection{Improved Dispatch of Stochastic Producers (ImpD)}

In an attempt to increase the performance of the conventional dispatch model (1), we address now the following question: Which value $p_{W}^{\max }$ should 
the forecast vector $\widehat{W}$ in $(1 \mathrm{~d})$ be replaced with to maximize market efficiency? The answer to this question is naturally given by the following bilevel programming problem:

$$
\begin{gathered}
\underset{p_{G}, p_{W}, \delta^{0}, p_{W}^{\max } ; y_{\omega}, \delta_{\omega}, \forall \omega}{\operatorname{Minimize}} \mathcal{C}^{\mathrm{D}}\left(p_{G}, p_{W}\right)+\mathbb{E}_{\omega}\left[\mathcal{C}^{\mathrm{B}}\left(y_{\omega}\right)\right] \\
\text { s.t. } \quad h^{\mathrm{B}}\left(y_{\omega}, \delta_{\omega}, \delta^{0}\right)+W_{\omega}-p_{W}=0, \forall \omega \in \Omega, \\
g^{\mathrm{B}}\left(y_{\omega}, \delta_{\omega}, p_{G} ; W_{\omega}\right) \leq 0, \forall \omega \in \Omega, \\
0 \leq p_{W}^{\max } \leq \bar{W}, \quad \\
\left(p_{G}, p_{W}, \delta^{0}\right) \in \arg \left\{\begin{array}{c}
\operatorname{Minimize}_{x_{G}, x_{W}, \theta} \quad \mathcal{C}^{\mathrm{D}}\left(x_{G}, x_{W}\right) \\
\text { s.t. } \quad h^{\mathrm{D}}\left(x_{G}, x_{W}, \theta\right)-l=0: \lambda^{\mathrm{D}}, \\
g^{\mathrm{D}}\left(x_{G}, \theta\right) \leq 0, \\
\left.x_{W} \leq p_{W}^{\max }\right\} .
\end{array}\right.
\end{gathered}
$$

The lower-level problem $(4 \mathrm{e})-(4 \mathrm{~h})$ is equivalent to the conventional dispatch (1), except for the upper bound of the day-ahead schedule of stochastic producers in $(4 \mathrm{~h})$, which is, in this case, endogenously computed by the upper-level problem $(4 \mathrm{a})-(4 \mathrm{~d})$ to minimize the sum of day-ahead dispatch costs and the expected balancing costs. Consequently, the bilevel model (4) manages to dispatch stochastic producers not only based on their marginal costs (which are often very low or zero), but also on the cost of their uncertainty (which is estimated by $(4 \mathrm{a})-(4 \mathrm{~d})$ ).

If the conventional dispatch model (1) is linear-note that this includes the family of dispatch models that consider piecewise linear supply costs functions, a DC power-flow network model, a piecewise linear approximation of the transmission losses, ramping constraints, etc. (see e.g. Motto et al. (2002)) - the lower-level problem (4e)-(4h) can be replaced by its KKT conditions. In turn, the associated complementarity conditions can be recast using the equivalent mixed-integer formulation proposed by Fortuny-Amat 
\& McCarl (1981). The steps required to transform a bilevel programming problem of the type of (4), with a linear lower level, into a manageable single-level optimization problem are well known in the technical literature (see e.g. Fernández-Blanco et al. (2012)) and are omitted here for conciseness. However, this transformation is illustrated later, in Section 3, using a small example.

For ease of comparison, the short form "ImpD" is used to refer to the conventional dispatch model (1) where $\widehat{W}$ in (1d) is replaced with the optimal value of $p_{W}^{\max }$ that results from (4).

\subsection{Energy-only Market Settlement}

We now introduce a standard settlement scheme whereby market participants are paid for energy only.

Consider a certain market participant $k$ and define $E_{k}^{\mathrm{D}}$ as the amount of energy sold (if positive) or purchased (if negative) in the day-ahead market, and $E_{k \omega^{\prime}}^{\mathrm{B}}$ as the amount of energy sold (if positive) or purchased (if negative) in the balancing market in scenario $\omega^{\prime}$. These quantities are directly derived from the power schedule that is solution to the dispatch model under consideration. The payment to (if positive) or from (if negative) market participant $k$ under scenario $\omega^{\prime}$ is then given by

$$
\lambda_{s(k)}^{\mathrm{D}} E_{k}^{\mathrm{D}}+\lambda_{s(k) \omega^{\prime}}^{\mathrm{B}} E_{k \omega^{\prime}}^{\mathrm{B}},
$$

where $s(k)$ indicates the node where market participant $k$ is located. The locational day-ahead market price $\lambda_{s(k)}^{\mathrm{D}}$ is obtained from either ConvD, StochD, or ImpD, while the locational balancing market price $\lambda_{s(k) \omega^{\prime}}^{\mathrm{B}}$ is computed from (2) after the day-ahead market is cleared and the actual realization $\omega^{\prime}$ of the stochastic production becomes known.

Morales et al. (2012) shows that, if generating units are fully dispatchable from zero to their maximum capacities (the problem of pricing in markets with non-convexities is not treated here; see e.g. Bjørndal \& Jörnsten (2008) 
for further information on this topic), the energy-only settlement scheme (5) under the stochastic dispatch model (3) guarantees cost recovery for flexible producers only in expectation. This expectation is, besides, contingent on the probabilistic characterization of the stochastic production at a marketwide level, which is in possession of the TSO and out of the control of the individual producers. Furthermore, we show in the illustrative example of Section 3 that StochD may actually dispatch flexible units in the day-ahead market in a loss-making position.

On the contrary, the conventional dispatch model, either in the traditional form of ConvD or in the variant ImpD proposed in this paper, ensures cost recovery for flexible producers for any possible realization of the stochastic production.

\section{Results and Discussion}

In this section, we first make use of a small two-node system to intuitively illustrate the main features of the previously discussed dispatch models. Then, we provide meaningful results from a more realistic case study.

\subsection{Illustrative Example}

The different dispatch models are illustrated next using the two-node system depicted in Fig. 1. This small system consists of one line, two loads ( $L_{1}$ and $L_{2}$ ), three conventional generators $\left(G_{1}, G_{2}\right.$, and $\left.G_{3}\right)$, and one wind power plant (WP). The capacity and reactance of the line are $100 \mathrm{MW}$ and $0.13 \mathrm{pu}$, respectively. Loads $L_{1}$ and $L_{2}$ are assumed to be inelastic and equal to 80 and $90 \mathrm{MW}$, respectively. The demand that is involuntarily shed is valued at $\$ 200 / \mathrm{MWh}$. The stochastic power output of the wind farm is modeled by two plausible scenarios, which are referred to as high (50 MW) and low (10 MW), with probabilities of occurrence equal to 0.6 and 0.4 .

Data for the conventional units are collated in Table 1 , where $\bar{P}$ is the unit capacity; $C$ is the price offer for energy sale in the day-ahead market; 


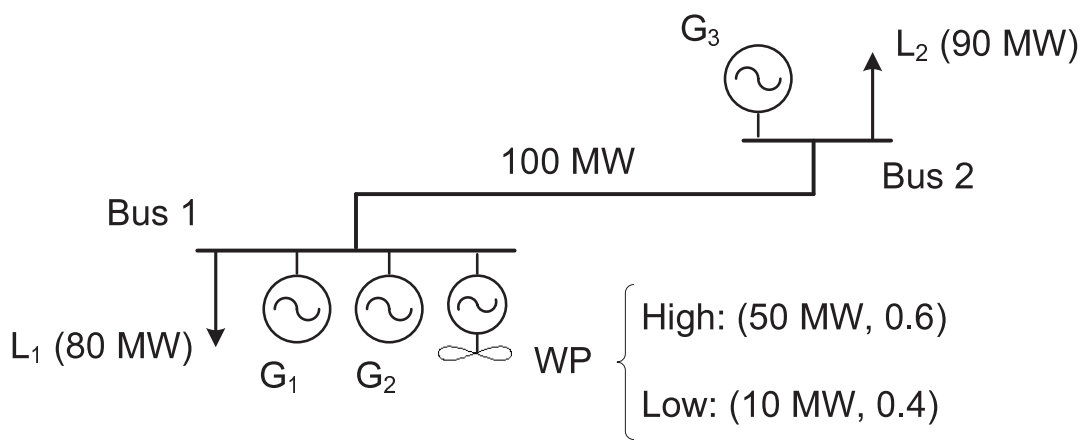

Figure 1: Two-bus power system.

Table 1: Unit data - Two-bus system

\begin{tabular}{lccc}
\hline Unit & $G_{1}$ & $G_{2}$ & $G_{3}$ \\
\hline $\bar{P}(\mathrm{MW})$ & 100 & 110 & 50 \\
$C(\$ / \mathrm{MWh})$ & 35 & 30 & 10 \\
$C^{+}(\$ / \mathrm{MWh})$ & 40 & - & - \\
$C^{-}(\$ / \mathrm{MWh})$ & 34 & - & - \\
$R^{+}(\mathrm{MW})$ & 20 & 0 & 0 \\
$R^{-}(\mathrm{MW})$ & 40 & 0 & 0 \\
\hline
\end{tabular}

$C^{+}$and $C^{-}$are, respectively, the price offers for energy sale and purchase in the balancing market; and $R^{+}$and $R^{-}$are, in that order, the upper bounds of the energy sale and purchase offers in the balancing market. Note that, in comparative terms, unit $G_{1}$ is expensive, but flexible; unit $G_{2}$ is a little bit cheaper, but inflexible; and unit $G_{3}$ is very cheap, but inflexible. Therefore, $G_{1}$ is the only unit in the system that can be re-dispatched to provide balancing energy. Besides, observe that, for this unit, $C^{+}>C$ and $C^{-}<C$, meaning that producer $G_{1}$ is willing to be flexible in return for a price premium on the energy traded during the balancing operation (Pritchard et al., 2010).

The marginal cost of the energy produced by the wind farm is considered 
to be zero. The expected wind power production is $50 \times 0.6+10 \times 0.4=$ 34 MW.

\subsubsection{Dispatch Models}

Firstly, we consider the conventional dispatch model (1), which writes for this particular example as follows:

$$
\begin{array}{ll}
\text { Min. } & 35 p_{G_{1}}+30 p_{G_{2}}+10 p_{G_{3}} \\
\text { s.t. } & p_{G_{1}}+p_{G_{2}}+p_{W}-80=-\frac{\delta_{2}^{0}}{0.13}, \\
& p_{G_{3}}-90=\frac{\delta_{2}^{0}}{0.13}, \\
& p_{G_{1}} \leq 100, \quad p_{G_{2}} \leq 110, \quad p_{G_{3}} \leq 50, \\
& -100 \leq \frac{\delta_{2}^{0}}{0.13} \leq 100, \\
& p_{W} \leq 34, \\
& p_{G_{1}}, p_{G_{2}}, p_{G_{3}}, p_{W} \geq 0,
\end{array}
$$

where bus 1 is considered as the reference node, i.e. $\delta_{1}^{0}=0$. Optimization problem (6) aims at minimizing the day-ahead production costs (6a). The dispatch problem is built upon a DC modeling of the transmission network, which leads to the set of nodal power balance equations (6b) and (6c), and includes generation and transmission capacity limits, (6d) and (6e), respectively. As it is customary, constraint (6f) limits the dispatch of the wind power plant to its expected production. Constraints $(6 \mathrm{~g})$ enforce the nonnegative character of production quantities.

Observe that, according to the dispatch model (6), the day-ahead market is settled irrespective of the potential impact that the resulting day-ahead program $\left\{p_{G_{1}}^{*}, p_{G_{2}}^{*}, p_{G_{3}}^{*}, p_{W}^{*}\right\}$ may have on the subsequent balancing operation. The day-ahead market is thus cleared purely based on a least-cost merit-order

principle. This way, the wind farm is first dispatched to $34 \mathrm{MW}$ (its expected production), followed by generating units $G_{3}$ and $G_{2}$, in that order, which 
are dispatched to 50 and $86 \mathrm{MW}$, respectively, to cover the total system load of $170 \mathrm{MW}$. Unit $G_{1}$ (the flexible producer) is consequently left out of the day-ahead schedule. Afterwards, during the balancing operation of the power system, energy adjustments to the day-ahead schedule are required to cope with the uncertain wind power production. Specifically, if the power output of the wind farm turns out to be high (50 MW), the wind power producer seeks to sell the leftover $50-34=16 \mathrm{MW}$ in this market. However, the only flexible unit in the system, unit $G_{1}$, cannot purchase the extra wind, as it cannot decrease its production below zero. As a result, these $16 \mathrm{MW}$ of free wind power have to be spilled. On the other hand, if the eventual wind generation is low $(10 \mathrm{MW})$, there is a wind generation deficit of $34-10=24 \mathrm{MW}$. This deficit has to be covered in the balancing market, but generating unit $G_{1}$ can only increase its production $20 \mathrm{MW}$ at most. Consequently, the remaining $24-20=4 \mathrm{MW}$ are obtained from costly load curtailment.

We can alternatively compute the day-ahead generation schedule using the stochastic dispatch model (3), which writes as follows:

$$
\begin{aligned}
\text { Min. } & 35 p_{G_{1}}+30 p_{G_{2}}+10 p_{G_{3}}+0.6\left(40 r_{G_{1} h}^{+}-34 r_{G_{1} h}^{-}+200\left(l_{1 h}^{\text {shed }}+l_{2 h}^{\text {shed }}\right)\right) \\
& +0.4\left(40 r_{G_{1} l}^{+}-34 r_{G_{1} l}^{-}+200\left(l_{1 l}^{\text {shed }}+l_{2 l}^{\text {shed }}\right)\right) \\
\text { s.t. } & (6 \mathrm{~b})-(6 \mathrm{e}),(6 \mathrm{~g}) \\
& p_{W} \leq 50 \\
& r_{G_{1} h}^{+}-r_{G_{1} h}^{-}+l_{1 h}^{\text {shed }}+50-p_{W}-W_{h}^{\text {spill }}=\frac{\left(\delta_{2}^{0}-\delta_{2 h}\right)}{0.13} \\
& r_{G_{1} l}^{+}-r_{G_{1} l}^{-}+l_{1 l}^{\text {shed }}+10-p_{W}-W_{l}^{\text {spill }}=\frac{\left(\delta_{2}^{0}-\delta_{2 l}\right)}{0.13} \\
& l_{2 h}^{\text {shed }}=-\frac{\left(\delta_{2}^{0}-\delta_{2 h}\right)}{0.13} \\
& l_{2 l}^{\text {shed }}=-\frac{\left(\delta_{2}^{0}-\delta_{2 l}\right)}{0.13}, \\
& p_{G_{1}}+r_{G_{1} h}^{+} \leq 100, p_{G_{1}}+r_{G_{1} l}^{+} \leq 100
\end{aligned}
$$


where subscripts " $h$ " and "l" index the corresponding augmented variable with scenario "high" and "low", respectively. Note that the cleared amount of wind production in the day-ahead market, $p_{W}$, is limited to its capacity (50 MW) through constraint (7c).

Optimization problem (7) includes the scenario-based modeling of the balancing operation through the set of constraints $(7 \mathrm{~d})-(7 \mathrm{o})$. Balancing actions comprise the production increase/decrease of flexible unit $G_{1}\left(r_{G_{1}}^{+} / r_{G_{1}}^{-}\right)$, wind spillage $\left(W^{\text {spill }}\right)$, and load shedding $\left(l_{1}^{\text {shed }}, l_{2}^{\text {shed }}\right)$. The stochastic dispatch model seeks to minimize the overall expected system costs (7a), which consists of the day-ahead dispatch costs plus the expectation of the balancing operation costs. Constraints $(7 \mathrm{~d})-(7 \mathrm{~g})$ enforce the power balances per node and scenario. Inequalities $(7 \mathrm{~h})-(7 \mathrm{j})$ impose generation and transmission capacity limits at the balancing stage. Constraints $(7 \mathrm{k})$ and (7l) limit the balancing energy provided by unit $G_{1}$ to its "flexible capacity", which is specified through $R^{+}$and $R^{-}$in Table 1 for production increases and decreases, respectively. Inequalities $(7 \mathrm{~m})$ and $(7 \mathrm{n})$ cap, in that order, the amount of wind power that is spilled and the amount of load that is shed to the actual wind power production and the actual load consumption. Finally, the set of constraints (7o) constitute positive variable declarations.

The essential feature of the stochastic dispatch model (7) is that the dayahead generation schedule $\left\{p_{G_{1}}, p_{G_{2}}, p_{G_{3}}, p_{W}\right\}$ is determined considering its 
projected implications for the subsequent balancing operation of the power system. Following this rationale, only $10 \mathrm{MW}$ of wind power production are cleared in the day-ahead market. Furthermore, the flexible, but expensive, generating unit $G_{1}$ is dispatched to $40 \mathrm{MW}$ in order to exploit its capability of reducing its power output during the balancing operation. Thus, if scenario high materializes, the 40-MW wind production surplus can be sold to unit $G_{1}$ instead of being curtailed. Besides, since the share of unit $G_{1}$ in the day-ahead schedule is increased up to $40 \mathrm{MW}$, unit $G_{2}$ is only dispatched to $70 \mathrm{MW}$, even though this unit is $\$ 5 / \mathrm{MWh}$ cheaper than unit $G_{1}$. Therefore, the least-cost merit-order principle that drives the conventional dispatch model is here violated.

We compute next the amount of wind power production that should clear the day-ahead market to maximize power system efficiency under the conventional dispatch model. For this purpose, we solve the following bilevel programming problem:

$$
\begin{aligned}
\text { Min. } & 35 p_{G_{1}}+30 p_{G_{2}}+10 p_{G_{3}}+0.6\left(40 r_{G_{1} h}^{+}-34 r_{G_{1} h}^{-}+200\left(l_{1 h}^{\text {shed }}+l_{2 h}^{\text {shed }}\right)\right) \\
& +0.4\left(40 r_{G_{1} l}^{+}-34 r_{G_{1} l}^{-}+200\left(l_{1 l}^{\text {shed }}+l_{2 l}^{\text {shed }}\right)\right) \\
\text { s.t. } & r_{G_{1} h}^{+}-r_{G_{1} h}^{-}+l_{1 h}^{\text {shed }}+50-p_{W}-W_{h}^{\text {spill }}=\frac{\left(\delta_{2}^{0}-\delta_{2 h}\right)}{0.13} \\
& r_{G_{1} l}^{+}-r_{G_{1} l}^{-}+l_{1 l}^{\text {shed }}+10-p_{W}-W_{l}^{\text {spill }}=\frac{\left(\delta_{2}^{0}-\delta_{2 l}\right)}{0.13} \\
& l_{2 h}^{\text {shed }}=-\frac{\left(\delta_{2}^{0}-\delta_{2 h}\right)}{0.13} \\
& l_{2 l}^{\text {shed }}=-\frac{\left(\delta_{2}^{0}-\delta_{2 l}\right)}{0.13} \\
& p_{G_{1}}+r_{G_{1} h}^{+} \leq 100, \quad p_{G_{1}}+r_{G_{1} l}^{+} \leq 100 \\
& p_{G_{1}}-r_{G_{1} h}^{-} \geq 0, p_{G_{1}}-r_{G_{1} l}^{-} \geq 0 \\
& -100 \leq \frac{\delta_{2 h}}{0.13} \leq 100,-100 \leq \frac{\delta_{2 l}}{0.13} \leq 100 \\
& r_{G_{1} h}^{+} \leq 20, \quad r_{G_{1} l}^{+} \leq 20,
\end{aligned}
$$


where the dual variables of the lower-level problem (8o)-(8u) have been made explicit after the corresponding constraint, separated by a colon.

Notice that $p_{W}^{\max }$ is a decision variable of the upper-level problem that enters the lower-level problem as a constant. This variable is limited to the capacity of the wind farm through constraint (8n). The remaining equations are the same as those in the conventional and stochastic dispatch models (6) and (7).

For the bilevel programming problem (8) to be processed by optimization solvers, it has to be first transformed into an equivalent single-level optimization problem. To this end, we can replace the lower-level minimization problem $(8 \mathrm{o})-(8 \mathrm{u})$ with its KKT conditions, which are as follows:

$$
35+\lambda_{1}^{\mathrm{D}}+\bar{\mu}_{G_{1}}-\underline{\mu}_{G_{1}}=0,
$$


Besides, the complementarity conditions (9h)-(9k) can be recast using the mixed-integer linear formulation introduced by Fortuny-Amat \& McCarl (1981). For example, consider a large enough constant $M$. The complementarity condition $(9 \mathrm{j})$ can be equivalently formulated as

$$
\begin{aligned}
\bar{\rho} & \leq M u \\
p_{W}^{\max }-p_{W} & \leq \bar{W}(1-u),
\end{aligned}
$$

where $u$ is a binary variable, i.e. $u \in\{0,1\}$, and $\bar{W}$ is the capacity of the wind farm, equal to $50 \mathrm{MW}$. Notice that both quantities in the left-hand side of the inequalities above must be nonnegative as a result of (9g) and (9l).

After all these transformations, the bilevel program (8) leads to a singlelevel mixed-integer linear programming problem that can be readily processed by off-the-shelf optimization software and results in $p_{W}^{\max *}=30 \mathrm{MW}$. Consequently, under ImpD (the conventional settlement with a smart day- 
Table 2: Comparison of expected system operation costs $(\$)$ - Two-bus system

\begin{tabular}{lcccc}
\hline & Total & Day ahead & Balancing & $\begin{array}{c}\text { Load } \\
\text { curtailment }\end{array}$ \\
\hline ConvD & 3720 & 3080 & 320 & 320 \\
StochD & 3184 & 4000 & -816 & 0 \\
ImpD & 3520 & 3200 & 320 & 0 \\
\hline
\end{tabular}

ahead dispatch of the wind farm), only $30 \mathrm{MW}$ of wind power production are cleared in the day-ahead market, which avoids expensive load curtailment if scenario low eventually realizes. The conventional units are cleared following a least-cost merit order. In particular, generating units G1, G2, and G3 are dispatched to 0, 90, and $50 \mathrm{MW}$, respectively. As a consequence, $20 \mathrm{MW}$ of wind power have to be spilled if scenario high realizes.

Table 2 provides the breakdown of the expected system operation cost under each dispatch model. Logically, both StochD and ImpD outperform ConvD. Observe, moreover, that both StochD and $\mathrm{ImpD}$ result in a more costly day-ahead dispatch, which leads, however, to savings in the balancing operation stage without load shedding. In fact, the stochastic dispatch model is able to reduce costs at the balancing operation phase through a more efficient integration of the wind production. However, the energy-only market settlement associated with this dispatch model requires the flexible producer $G_{1}$ to accept economic losses if scenario low comes true, as we show in the following section.

\subsubsection{Prices and Revenues}

Energy prices resulting from each of the dispatch models are shown in Table 3. Note that these prices do not differ between buses, because no network congestion occurs in any of the two wind power scenarios considered. Observe that, for the three dispatch models, the resulting day-ahead electricity price is $\$ 30 / \mathrm{MWh}$, which is the marginal cost of unit $G_{2}$. In the 
Table 3: Day-ahead and balancing energy prices (\$/MWh)-Two-bus system

\begin{tabular}{lccc}
\hline & $\lambda_{n}^{\mathrm{D}}, \forall n \in N$ & \multicolumn{2}{c}{$\lambda_{n \omega}^{\mathrm{B}}, \forall n \in N$} \\
\cline { 3 - 4 } & & High & Low \\
\hline ConvD & 30 & 0 & 200 \\
StochD & 30 & 25.67 & 36.50 \\
ImpD & 30 & 0 & 75 \\
\hline
\end{tabular}

case of ConvD, the value of lost load ( $\$ 200 / \mathrm{MWh})$ determines the balancing energy price in scenario low, where load shedding actions need to be undertaken if the day-ahead generation schedule given by this dispatch model is implemented. In both ConvD and ImpD, the balancing electricity price is set to $\$ 0 / \mathrm{MWh}$ in scenario high due to the occurrence of wind curtailment.

Given the energy prices in Table 3 and the dispatch results previously discussed, we can determine the profit made by each market participant in expectation and per scenario according to each dispatch model (see Table 4). For example, the payment to the flexible generator $G_{1}$ in scenario low under StochD is given by $40 \times 30=\$ 1200$. Since its marginal cost is equal to $\$ 35 / \mathrm{MWh}$, the profit that generator $G_{1}$ makes in this scenario is equal to $1200-40 \times 35=-\$ 200$. Here we bump into one of the most controversial features of StochD, namely, the likelihood that flexible units incur economic losses in some scenarios, even though the recovery of costs is guaranteed in expectation. Actually, notice that unit $G_{1}$ enters the day-ahead dispatch in a loss-making position, because its marginal cost, $\$ 35 / \mathrm{MWh}$, is higher than the resulting day-ahead market price, $\$ 30 / \mathrm{MWh}$. Therefore, under StochD, being flexible may involve higher risk than being inflexible, which may potentially discourage power producers from providing balancing service. In contrast, ConvD and the proposed ImpD ensure revenue adequacy in the day-ahead market and per scenario, and therefore they do not suffer from this problem. 
Table 4: Profit (\$) of market participants-Two-bus system

\begin{tabular}{rrrrr}
\hline & & & \multicolumn{2}{c}{ Per scenario } \\
\cline { 3 - 5 } & & & High & Low \\
\hline \multirow{4}{*}{ ConvD } & Expected & & & \\
& $G_{1}$ & 1320 & 0 & 3300 \\
& $G_{2}$ & 0 & 0 & 0 \\
& $G_{3}$ & 1000 & 1000 & 1000 \\
& $\mathrm{WP}$ & -900 & 1020 & -3780 \\
& $L_{1}$ & -2400 & -2400 & -2400 \\
& $L_{2}$ & -2380 & -2700 & -1900 \\
\hline \multirow{4}{*}{ StochD } & $G_{1}$ & 24 & 173.33 & -200 \\
& $G_{2}$ & 0 & 0 & 0 \\
& $G_{3}$ & 1000 & 1000 & 1000 \\
& $\mathrm{WP}$ & 916 & 1326.66 & 300 \\
& $L_{1}$ & -2400 & -2400 & -2400 \\
& $L_{2}$ & -2700 & -2700 & -2700 \\
\hline \multirow{6}{*}{ ImpD } & $G_{1}$ & 320 & 0 & 800 \\
& $G_{2}$ & 0 & 0 & 0 \\
& $G_{3}$ & 1000 & 1000 & 1000 \\
& $\mathrm{WP}_{1}$ & 300 & 900 & -600 \\
& $L_{1}$ & -2400 & -2400 & -2400 \\
& $L_{2}$ & -2700 & -2700 & -2700 \\
\hline & & & &
\end{tabular}


Table 5: Generator data $(*=\{+,-\})$. Powers in MW

\begin{tabular}{ccccc}
\hline Unit & Type & Bus \# & $\bar{P}$ & $R^{*}$ \\
\hline 1 & $\mathrm{U} 76$ & 1 & 152 & 40 \\
2 & $\mathrm{U} 76$ & 2 & 152 & 40 \\
3 & $\mathrm{U} 100$ & 7 & 300 & 70 \\
4 & $\mathrm{U} 197$ & 13 & 591 & 180 \\
5 & $\mathrm{U} 12$ & 15 & 60 & 60 \\
6 & $\mathrm{U} 155$ & 15 & 155 & 30 \\
7 & $\mathrm{U} 155$ & 16 & 155 & 30 \\
8 & $\mathrm{U} 400$ & 18 & 400 & 0 \\
9 & $\mathrm{U} 400$ & 21 & 400 & 0 \\
10 & $\mathrm{U} 50$ & 22 & 300 & 0 \\
11 & $\mathrm{U} 155$ & 23 & 310 & 60 \\
12 & $\mathrm{U} 350$ & 23 & 350 & 40 \\
\hline
\end{tabular}

\subsection{Case Study}

We now consider a 24-bus power system that is based on the single-area version of the IEEE Reliability Test System (Grigg et al., 1999). It includes 34 lines, 17 loads, and 12 generating units. The nodal location, type, capacity, and flexibility parameters of these units are collated in Table 5. Energy offers submitted by power producers in the day-ahead market consist of the four incremental cost/power blocks listed in Table 9 of Grigg et al. (1999), assuming the fuel costs used by Bouffard et al. (2005). We consider that nuclear and hydro power producers offer their production at zero price. Price premiums of $5 \%$ and $4 \%$ are assumed for the energy sold and purchased, respectively, in the balancing market. This means that flexible producers are willing to sell (purchase) energy in the balancing market at a price $5 \%$ higher (4\% lower) than their energy offer price in the day-ahead market. Nuclear and hydro units are assumed to be inflexible and therefore, they do not provide balancing energy.

Two wind farms are located at nodes 5 and 7 . The per-unit power pro- 
duction of these wind farms is modeled using Beta distributions, as in Fabbri et al. (2005). The shape parameters of these Beta distributions, denoted by $(\alpha, \beta)$, are equal to $(0.71,0.08)$ and $(3.78,1.62)$, respectively. Thus, the perunit forecast power outputs of the wind farms at nodes 5 and 7 are 0.9 and 0.7, in that order. Furthermore, the power outputs of both wind farms are assumed to be correlated with a correlation coefficient $\rho$. Correlated samples from the previous Beta distributions are obtained using the sampling procedure described by Morales et al. (2011). An original scenario set comprising 10000 wind power samples is first generated and then reduced to 100 using the fast forward selection algorithm presented by Heitsch \& Römisch (2003). The marginal costs of the wind farms are assumed to be zero.

Loads are considered to be inelastic with a value of lost load equal to $\$ 1000 /$ MWh. These loads are geographically distributed among buses as indicated in Table 5 of Grigg et al. (1999). The total system demand is $2000 \mathrm{MW}$. The capacities of lines 1-5, 5-10, and 7-8 are doubled (up to $350 \mathrm{MW}$ ) so that higher amounts of wind power production can be injected at buses 5 and 7 .

The single-level mixed-integer linear programming problem that results from the bilevel program (4) has been solved using CPLEX 12.3.0 under GAMS on a Windows-based personal computer Intel(R) Core(TM) i5 with four processors clocking at $2.4 \mathrm{GHz}$ and $6 \mathrm{~GB}$ of RAM. Solution time is kept below 30 seconds in all instances.

The stochastic dispatch model (3) has, among others, two properties that make it particularly useful to facilitate the large-scale integration of stochastic production in electricity markets, namely, its ability to avoid the uneconomic scheduling of stochastic production capacity and its ability to efficiently accommodate generation from stochastic producers that are spatially correlated. We show below that these two properties are conferred, to a large extent, on the conventional dispatch model (1), if solved for an appropriate value of stochastic production, generally different from the mean. This is 
actually what we refer to as ImpD.

Figure 2 shows the expected cost of the power system operation as a function of the wind power penetration level, for the three dispatch models. The wind power penetration level is defined as the ratio of the forecast wind power production to the total system demand and is increased by augmenting the capacity installed at both wind farms by the same amount. The figure is arranged in two illustrations, each corresponding to a different correlation coefficient between wind farms. Observe that from a certain penetration level, the expected cost resulting from the implementation of the conventional dispatch begins to significantly diverge from the expected cost yielded by the other two dispatch models. Furthermore, note that this "breaking point", roughly identified on the graphs using a vertical dashed line, occurs for lower penetration levels if the correlation coefficient between wind sites increases. Indeed, the breaking point moves approximately from $38 \%$ to $33 \%$ if the correlation coefficient goes from 0.35 to 0.75. In contrast, StochD and ImpD are significantly less affected by correlated winds, as they both account for the wind production variability to decide the wind generation schedule. Furthermore, notice that, in the case of ConvD, the expected cost exhibits an increasing trend after a high enough wind power penetration level, whereas both StochD and ImpD guarantee that an increase in wind power capacity never leads to an increase in the expected cost.

We now show that, unlike ConvD or ImpD, the stochastic dispatch leads to a conflicting energy-only settlement of the market, because it requires flexible producers to incur losses in some scenarios. Let us consider a wind power penetration level of $38 \%$. In this instance, generators 1, 2, 6, 7, 11 and 12 are mostly the units providing balancing energy. Table 6 includes the expected profit made by some of these units in these conditions under the three dispatch models. For the case of StochD, the average losses incurred by the selected units and the probability of their profit being eventually negative are also shown. Note that this probability is remarkably high. 
Figure 2: Impact of the wind power penetration level and spatial correlation on the expected cost of the system operation. Total system demand $=2000 \mathrm{MW}$. 
Table 6: Highlights of profits. Wind penetration $38 \%(\rho=0.35)$

\begin{tabular}{|c|c|c|c|c|c|}
\hline & & \multicolumn{4}{|c|}{ Unit } \\
\hline & & 1 & 6 & 11 & 12 \\
\hline \multirow[t]{2}{*}{ ConvD } & $\begin{array}{l}\text { Expected } \\
\text { profit }(\$)\end{array}$ & 379.8 & 359.7 & 724.9 & 389.1 \\
\hline & $\begin{array}{l}\text { Expected } \\
\text { profit }(\$)\end{array}$ & 45.6 & 48.4 & 99.7 & 64.9 \\
\hline \multirow[t]{2}{*}{ StochD } & $\begin{array}{l}\text { Average } \\
\text { losses (\$) }\end{array}$ & -17.4 & -10.9 & -17.6 & -11.5 \\
\hline & $\begin{array}{l}\text { Probability } \\
\text { profit }<0\end{array}$ & 0.81 & 0.71 & 0.71 & 0.75 \\
\hline $\operatorname{ImpD}$ & $\begin{array}{l}\text { Expected } \\
\text { profit }(\$)\end{array}$ & 170.2 & 263.7 & 531.6 & 178.7 \\
\hline
\end{tabular}

Lastly, observe that the expected profit made by the selected units is significantly higher under ConvD than under ImpD. This is so because, under the conventional dispatch where the expected wind power production is cleared, there is a considerable transfer of money from the wind power producers to the flexible producers, as the wind power producers have to bear the cost of a very inefficient balancing operation. ImpD manages to substantially mitigate this effect by clearing an amount of wind power production-not necessarily equal to the mean - that avoids high balancing costs.

\section{Conclusions}

This paper deals with the clearing of a day-ahead electricity market that includes a significant number of stochastic producers. Our study uses two reference models for generation scheduling: on the one hand, a conventional network-constrained auction based on a least-cost merit order for dispatch, where stochastic generation enters with its expected production and a very 
low marginal cost; on the other, a full stochastic dispatch method that maximizes market efficiency by anticipating balancing costs. The conventional dispatch may turn out to be very uneconomical, while the stochastic one leads to an energy-only market settlement that does not guarantee cost recovery for flexible producers is some scenarios.

We show that the conventional auction, if cleared with an appropriate value of stochastic production, generally different from the mean, can significantly approach the stochastic dispatch ideal. We construct a bilevel program that optimally computes this value. Our analysis prompts two fundamental conclusions, namely:

1. Current day-ahead markets should not clear the expected stochastic production by default. There is indeed room for substantial improvement in market efficiency by abandoning this practice, in particular in those markets with a high share of stochastic generation.

2. The amount of stochastic production to be cleared in the day-ahead market should be driven not only by the marginal cost of stochastic generation, which is usually very low or zero, but also by the cost of its uncertainty, understood as its economic impact due to system balancing.

As future research, it is necessary to develop computationally efficient methods that allow us to determine a day-ahead schedule for stochastic producers better in terms of market efficiency than their expected power outputs without having to directly solve a computationally costly bilevel program. Likewise, the idea introduced in this paper is compatible with the implementation of reserve capacity markets or the flexible ramping products that are currently under development in CAISO (Abdul-Rahman et al., 2012) and Midwest ISO (Navid \& Rosenwald, 2012). The combination of these strategies may bring current market efficiency closer to the full stochastic optimization ideal. 


\section{References}

Abdul-Rahman, K., Alarian, H., Rothleder, M., Ristanovic, P., Vesovic, B., \& Lu, B. (2012). Enhanced system reliability using flexible ramp constraint in CAISO market. In Power and Energy Society General Meeting, 2012 IEEE (pp. 1-6).

Birge, J. R., \& Louveaux, F. (2011). Introduction to Stochastic Programming. Series in Operations Research and Financial Engineering (2nd ed.). Springer.

Bjørndal, M., \& Jörnsten, K. (2008). Equilibrium prices supported by dual price functions in markets with non-convexities. European Journal of Operational Research, 190, 768-789.

Bouffard, F., \& Galiana, F. D. (2008). Stochastic security for operations planning with significant wind power generation. IEEE Transactions on Power Systems, 23, 306-316.

Bouffard, F., Galiana, F. D., \& Conejo, A. J. (2005). Market-clearing with stochastic security-Part II: case studies. IEEE Transactions on Power Systems, 20, 1827-1835.

Doherty, R., \& O’Malley, M. (2005). A new approach to quantify reserve demand in systems with significant installed wind capacity. IEEE Transactions on Power Systems, 20, 587-595.

Ela, E., Milligan, M., \& Kirby, B. (2011). Operating Reserves and Variable Generation. Technical Report NREL/TP-5500-51978 National Renewable Energy Laboratory.

Fabbri, A., Gomez San Roman, T., Rivier Abbad, J., \& Mendez Quezada, V. (2005). Assessment of the cost associated with wind generation prediction errors in a liberalized electricity market. IEEE Transactions on Power Systems, 20, 1440-1446. 
Fernández-Blanco, R., Arroyo, J., \& Alguacil, N. (2012). A unified bilevel programming framework for price-based market clearing under marginal pricing. IEEE Transactions on Power Systems, 27, 517-525.

Fortuny-Amat, J., \& McCarl, B. (1981). A representation and economic interpretation of a two-level programming problem. Journal of the Operational Research Society, (pp. 783-792).

Galiana, F. D., Bouffard, F., Arroyo, J. M., \& Restrepo, J. F. (2005). Scheduling and pricing of coupled energy and primary, secondary, and tertiary reserves. Proceedings of the IEEE, 93, 1970-1983.

Grigg, C., Wong, P., Albrecht, P., Allan, R., Bhavaraju, M., Billinton, R., Chen, Q., Fong, C., Haddad, S., Kuruganty, S. et al. (1999). The IEEE reliability test system-1996. IEEE Transactions on Power Systems, 14, 1010-1020.

Heitsch, H., \& Römisch, W. (2003). Scenario reduction algorithms in stochastic programming. Computational Optimization and Applications, 24, 187206.

Helman, U., Loutan, C., Rosenblum, G., Rothleder, M., Xie, J., Zhou, H., \& Kuo, M. (2010). Integration of Renewable Resources: Operational Requirements and Generation Fleet Capability at 20\% RPS. Technical Report California Independent System Operator (CAISO).

Hogan, W. W. (2005). On an Energy Only Electricity Market Design for Resource Adequacy. Technical Report Center for Business and Government, Harvard University.

Holttinen, H. (2005). Impact of hourly wind power variations on the system operation in the Nordic countries. Wind Energy, 8, 197-218. 
Morales, J. M., Conejo, A., Liu, K., \& Zhong, J. (2012). Pricing electricity in pools with wind producers. IEEE Transactions on Power Systems, 27, 1366-1376.

Morales, J. M., Conejo, A., \& Pérez-Ruiz, J. (2009). Economic valuation of reserves in power systems with high penetration of wind power. IEEE Transactions on Power Systems, 24, 900-910.

Morales, J. M., Conejo, A., \& Pérez-Ruiz, J. (2011). Simulating the impact of wind production on locational marginal prices. IEEE Transactions on Power Systems, 26, 820-828.

Motto, A., Galiana, F., Conejo, A., \& Arroyo, J. (2002). Network-constrained multiperiod auction for a pool-based electricity market. IEEE Transactions on Power Systems, 17, 646-653.

Navid, N., \& Rosenwald, G. (2012). Market solutions for managing ramp flexibility with high penetration of renewable resource. IEEE Transactions on Sustainable Energy, 3, 784-790.

Papavasiliou, A., Oren, S., \& O'Neill, R. (2011). Reserve requirements for wind power integration: A scenario-based stochastic programming framework. IEEE Transactions on Power Systems, 26, 2197-2206.

Pritchard, G., Zakeri, G., \& Philpott, A. (2010). A single-settlement, energyonly electric power market for unpredictable and intermittent participants. Operations Research, 58, 1210-1219.

Wong, S., \& Fuller, J. D. (2007). Pricing energy and reserves using stochastic optimization in an alternative electricity market. IEEE Transactions on Power Systems, 22, 631-638. 